Tropical Journal of Pharmaceutical Research January 2019; 18 (1): 25-30

ISSN: $1596-5996$ (print); 1596-9827 (electronic)

(c) Pharmacotherapy Group, Faculty of Pharmacy, University of Benin, Benin City, 300001 Nigeria.

Available online at http://www.tjpr.org

Original Research Article

http://dx.doi.org/10.4314/tjpr.v18i1.4

\title{
MiR-126 enhances cisplatin chemosensitivity in hepatocellular carcinoma cells by targeting IRS1
}

\author{
Jian Zuo, Rui Luo, Chenjie Huang, Xiaobin Lou, Lanjuan Li ${ }^{*}$ \\ State Key Laboratory for Diagnosis and Treatment of Infectious Diseases, Collaborative Innovation Center for Diagnosis \\ and Treatment of Infectious Diseases, The First Affiliated Hospital College of Medicine, Zhejiang University,Hangzhou310003, \\ China
}

*For correspondence: Email: Ijli@zju.edu.cn

Sent for review: 27 June 2018

Revised accepted: 15 December 2018

\begin{abstract}
Purpose: To investigate the potential role of miR-126 in regulating the proliferation and cisplatin chemosensitivity of human hepatocellular carcinoma (HCC) cells.

Methods: The expression of miR-126 was evaluated using clinical HCC specimens. MiR-126-mediated downregulation of Insulin Receptor Substrate 1 (IRS1) was determined by qRT-PCR, western blot and luciferase reporter assay. Cell Counting Kit-8 (CCK8) and colony formation assays were performed to examine the proliferation of HCC cells.

Results: Decreased expression of miR126 was found in HCC tumors and was correlated with poor survival in HCC patients. In HCC cells, miR-126 targeted IRS1 for downregulation, through which miR126 suppressed the growth of HCC cells and desensitized HCC cells to cisplatin treatment.

Conclusion: MiR-126a impairs the proliferation and cisplatin chemoresistance of HCC cells by targeting IRS1.
\end{abstract}

Keywords: miR-126, IRS1, HCC, cisplatin, chemosensitivity

\begin{abstract}
This is an Open Access article that uses a funding model which does not charge readers or their institutions for access and distributed under the terms of the Creative Commons Attribution License (http://creativecommons.org/licenses/by/4.0) and the Budapest Open Access Initiative (http://www.budapestopenaccessinitiative.org/read), which permit unrestricted use, distribution, and reproduction in any medium, provided the original work is properly credited.

Tropical Journal of Pharmaceutical Research is indexed by Science Citation Index (SciSearch), Scopus, International Pharmaceutical Abstract, Chemical Abstracts, Embase, Index Copernicus, EBSCO, African Index Medicus, JournalSeek, Journal Citation Reports/Science Edition, Directory of Open Access Journals (DOAJ), African Journal Online, Bioline International, Open-J-Gate and Pharmacy Abstracts
\end{abstract}

\section{INTRODUCTION}

Hepatocellular carcinoma (HCC) represents the most common type of liver cancer with approximately 800,000 newly diagnosed HCC cases annually worldwide [1]. Although chemotherapy has been proven to improve clinical benefits for HCC patients, the development of chemoresistance largely restrains the clinical efficacy of HCC chemotherapy [2]. Cisplatin, owing to its ability in suppressing tumor growth and inducing tumor cell apoptosis $[3,4]$, is widely used in the treatment of multiple kinds of cancers, including HCC [5].Unfortunately, a large proportion of HCC patients can develop cisplatin chemoresistance which finally leads to the failure of HCC chemotherapy [6]. However, the detailed molecular mechanisms underlying cisplatin chemoresistance are still elusive and need to be further elucidated.

MicroRNAs (miRNAs) are small (19 25 nucleotides in length), non-coding RNAs which bind to the untranslated region (UTR) of target mRNAs, leading to their degradation or 
transcriptional repression [7,8]. Accumulating evidence shave indicated that miRNAs are crucial mediators in regulating tumor growth and chemoresistence $[9,10]$. A previous study has linked reduced miR-126 expression to the metastatic recurrence of HCC [11].However, whether and how miR-126 participates in modulating the progression and cisplatin chemoresistance of $\mathrm{HCC}$ has not yet been explored to date. In this study, reduced expression of miR126 was observed in HCC tumors and was correlated with poor survival in HCC patients. Mechanistically, miR-126 caused growth inhibition of HCC cells and desensitized HCC cells to cisplatin treatment by downregulating the expression of IRS1, which reportedly has an oncogenic role in liver cancer. The present study identify miR-126 as a potential tumor suppressor, and can hopefully be beneficial to the clinical diagnosis or treatment of HCC.

\section{EXPERIMENTAL}

\section{Patients}

Tissue samples, including paired tumour and adjacent normal tissues, were collected from 30 HCC patients in The First Affiliated Hospital, College of Medicine, Zhejiang University, between January 2017 and June 2017. The median age of the HCC patients was 48.2 years (20 65 years). Informed consent was obtained from all participants, and this study was approved by the Ethics Committee of The First Affiliated Hospital, College of Medicine, Zhejiang University (No.2015-188), all procedures were performed in accordance with the World Medical Association Declaration of Helsinki [12].

\section{Cell lines}

Normal human liver cell line L-02 and HCC cell lines (SMMC-7721, Hep3B, HepG2, Huh7, MHCC-97L, MHCC-97H, and HCCLM3) were purchased from Shanghai Cell Bank of the Chinese Academy of Sciences (Shanghai, China). Cells were cultured in Dulbecco's Modified Eagle medium (DMEM) (HyClone, Logan, UT, USA) supplemented with $10 \%$ fetal bovine serum (FBS; Gibco, Gaithersburg, MD, USA), and $1 \%$ penicillin/streptomycin (Gibco) at $37^{\circ} \mathrm{C}$ in a $5 \% \mathrm{CO}_{2}$ humidified atmosphere.

\section{Plasmid construction and transfection}

MiR-126 mimics, control mimics, siRNA for IRS1 (si-IRS1), and control siRNA (si-NC) were purchased from GenePharma Inc. (Shanghai, China). IRS1 expression plasmid and control plasmid were purchased from Genechem (Shanghai, China). MiRNA or siRNA transfection were carried out using Lipofectamine 3000 reagent (Invitrogen, Waltham, MA, USA). $48 \mathrm{~h}$ after transfection, cells were harvested for further analysis.

\section{Quantitative PCR}

Total RNA was isolated using TRIzol reagent (Sigma-Aldrich, St. Louis, MO, USA). The cDNA was synthesized using a PrimeScript RT Reagent Kit with gDNA Eraser (Takara, Shiga, Japan) according to the manufacturer's instructions. Quantitative real-time polymerase chain reaction (qRT-PCR) was subsequently performed with SYBR Green Master Mix (Toyobo, Tokyo, Japan) using the primers listed in Table 1.The relative expression of miR-126 was normalized to $\mathrm{U} 6$ and the relative expression of IRS1 was normalized to GAPDH.

Table 1: Primers used in qRT-PCR

\begin{tabular}{ll}
\hline miR-126 forward & 5'-ACACTCCAGCTGGGTCGTACCGTGAGTAAT-3' \\
miR-126 reverse & 5'-TGGTGTCGTGGAGTCG-3' \\
U6 forward & 5'-GCTTCGGCAGCACATATACAAAAT-3' \\
U6 reverse & 5'-CGCTTCACGAATTTGCGTGTCAT-3' \\
IRS1 forward & 5'-CAACTGGACATCACAGCAGAA-3' \\
IRS1 reverse & 5'-ACTGAAATGGATGCATCGTACC-3' \\
GAPDH forward & 5'-TTTGTCAAGCTCATTTCCTG-3' \\
GAPDH reverse & 5'-TGGTCCAGGGTTTCTTACTC-3'
\end{tabular}

\section{Western blot}

Whole-cell protein extracts were prepared using radioimmunoprecipitation assay (RIPA) buffer (EMB Millipore, Hayward, CA, USA). The total protein concentration was determined using a bicinchoninic acid (BCA) assay kit (Thermo Scientific, Scotts Valley, CA, USA).20 40 $\mu \mathrm{g}$ protein samples were electrophoresed and then were transferred onto polyvinylidene difluoride (PVDF) membranes (Millipore). The membranes were blocked in 5\% non-fat milk and incubated overnight at $4^{\circ} \mathrm{C}$ with primary antibodies against IRS1 (1:500, ab52167; Abcam, Burlingame, CA, USA)followed by incubation with horseradish peroxidase (HRP)-conjugated secondary antibody.

\section{Luciferase reporter assay}

The cDNA fragments from IRS1 containing the wild-type (wt) untranslated region (UTR) (IRS13'-UTR-wt) or mutant (mut)-type (IRS1-3'-UTRmut) miR-126 binding sites were cloned into a pGL3 vector (Promega, Sunnyvale, CA, USA). Firefly and renilla luciferase activities were analysed with a Dual Luciferase Assay Kit (Promega). 


\section{Colony formation assay}

Cells were treated with $10 \mu \mathrm{M}$ cisplatin for 2 days followed by three washes with PBS, and then cultured in cisplatin-free media for 10 days. Cell colonies were fixed with methanol and stained with crystal violet (Sigma-Aldrich).

\section{Statistical analysis}

All data are presented as mean \pm SEM. Statistical analysis was performed using GraphPad Prism software using Student's $t$-tests or log-rank test. $P<0.05$ was considered statistically significant.

\section{RESULTS}

\section{MiR-126 expression is downregulated in HCC patients}

First, miR-126 expression was assessed in 30 paired HCC tumors and adjacent normal tissues. QRT-PCR results demonstrated significantly reduced miR-126 expression in HCC tumors compared to adjacent normal tissues (Figure 1 a). Furthermore, we also found significantly higher miR-126 expression in normal human liver cell line L-02 than that in HCC cell lines, including SMMC-7721, Hep3B, HepG2, Huh7, MHCC-97L, HCCLM3, and MHCC-97H cells(Figure 1 b). This finding was further reinforced by data-mining using TCGA datasets in which the expression of miR-126 also showed a significant reduction in HCC cohort compared to control cohort (Figure $1 \mathrm{c}$ ). More importantly, low miR-126 expression predicted poor overall survival in HCC patients (Figure $1 \mathrm{~d}$ ). These findings indicate that miR-126 may function as a tumor suppressor in HCC.

\section{MiR-126 represses the expression of IRS1}

To mechanistically determine the role of miR-126 in HCC development and progression, we predicted the target genes of miR-126 using the bioinformatics algorithm TargetScan website. MiR-126 has a putative binding site from 106 112 bp within the $3^{\prime}$-UTR region of IRS1 gene (Figure $2 \mathrm{a}$ ), whose product (IRS1 protein) was reported to promote tumor growth and chemoresistance [13-15]. To verify this speculation, we transfected miR-126 mimics into two HCC cell lines HCCLM3 and MHCC-97H, and found that both mRNA and protein levels of IRS1 were significantly inhibited upon miR-126 overexpression (Figure $2 \mathrm{~b}$ and $\mathrm{c}$ ). In luciferase reporter assay, miR-126 overexpression significantly reduced the luciferase activity in HCC cells transfected with IRS1-wt reporter but not in those transfected with IRS1-mut reporter, which harbors a mutant binding site for miR126(Figure $2 \mathrm{~d}$ ). Moreover, mRNA abundance of IRS1 was significantly higher in HCC tumors than that in adjacent normal tissues (Figure 2e). Importantly, there was a significant negative correlation between the levels of miR-126 and IRS1 in HCC patients (Figure $2 \mathrm{f}$ ). Similarly, IRS1 expression was significantly higher in HCC cell lines than in normal liver cell line (Figure $2 \mathrm{f}$ ), and a negative correlation between the levels of miR126 and IRS-1 was also observed in these cell lines (Figure $2 \mathrm{~g}$ ). Thus, miR-126 dampens IRS1 expression in HCC cells.

\section{Overexpression of miR-126 suppresses HCC cell proliferation}

To determine the functional impacts of miR-126 and IRS1 in HCC cells, we transfected HCCLM3 and MHCC-97H cells with miR-126 mimics to restore miR-126 expression. Forced expression of miR-126 significantly inhibited the proliferation of HCC cells as evaluated by CCK-8 assay (Figure $3 a, b)$. On the other hand, silencing the expression of IRS1 in HCC cells also caused significant growth inhibition of HCC cells (Figure $3 \mathrm{c}, \mathrm{d}$ ). These results suggest that IRS1 facilitates the proliferation of HCC cells, whereas miR-126 causes the opposite effect.

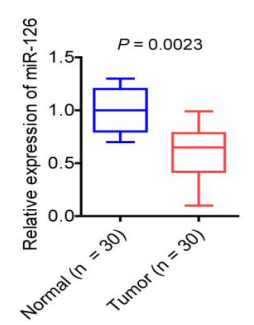

C

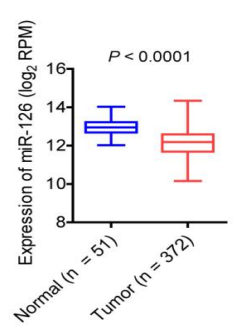

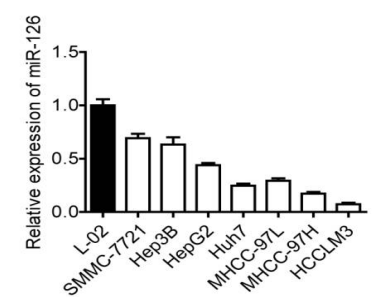

d

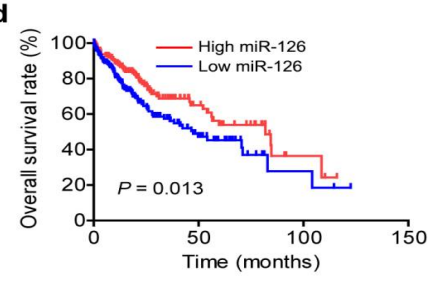

Figure 1: The expression of miR-126 is decreased in HCC tumors. (a) MiR-126 level in HCC tumors and tumor adjacent tissues was determined by qRT-PCR. (b) MiR-126 level in normal human liver cell line L-02 and HCC cell lines was determined by qRT-PCR. (c) MiR-126 level was analyzed using TCGA database. (d) The survival of HCC patients with high or low miR126 expression was analyzed using TCGA database. Data are shown as the mean \pm SEM; $p<0.05,{ }^{* *} p<$ $0.01,{ }^{* * *} p<0.001$ 


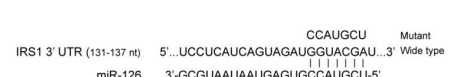

miR-126 3-GCGUAAUAAUgagugCCAUGCU-5

b
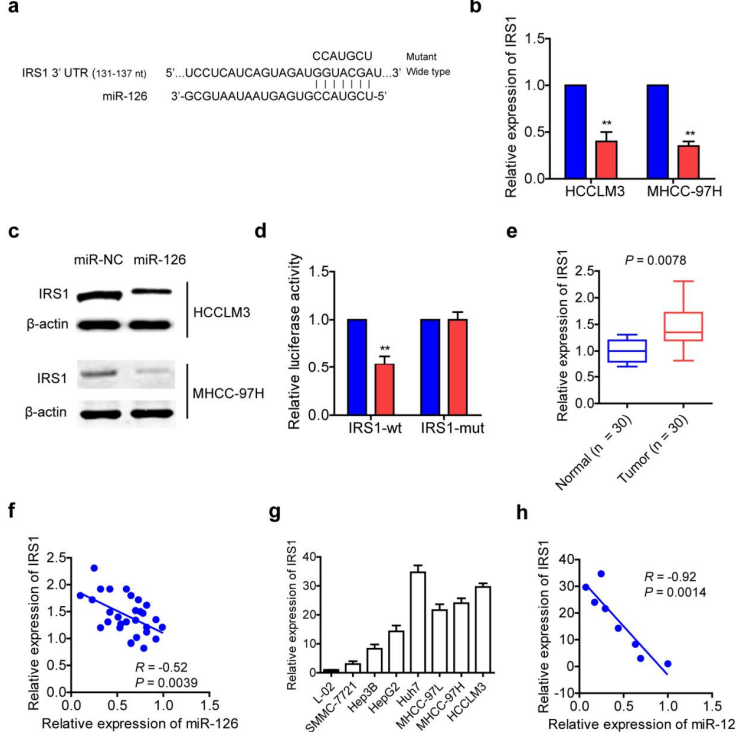

Figure 2: MiR-126 targets IRS1 for downregulation. (a) Sequence alignment of miR-126 and 3'-UTR region of humanIRS1 gene. (b, c) The mRNA (b) and protein (c) levels of IRS1 in miR-126-transfected or shamtransfected HCC cells was determined by qRT-PCR and western blot, respectively. (d) Luciferase activity was measured in HCCLM3 cells transfected with wtormut-IRS1 luciferase reporter constructs. (e) The mRNA level of IRS1 in HCC tumors and tumoradjacent tissues was analysed by qRT-PCR. (f) The mRNA level of IRS1 in L-02 cells and various HCC cell lines was determined by qRT-PCR. (g) Association between the levels of miR-126 and IRS1 in HCC tumors was evaluated by Spearman's rank correlation test. (h) Association between the levels of miR-126 and IRS1 in different liver cell lines was evaluated by Spearman's rank correlation test. Data are shown as the mean \pm SEM. $\left({ }^{*} p<0.05,{ }^{* *} p<0.01,{ }^{* \star *} p<0.001\right)$

a
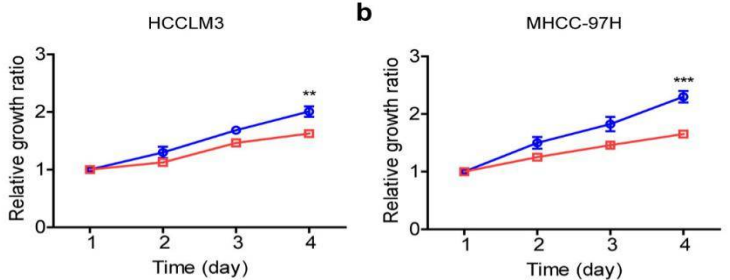

c

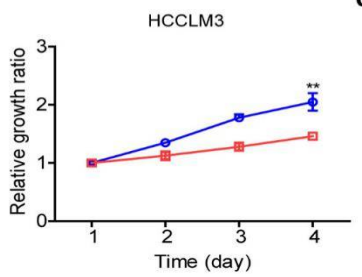

d

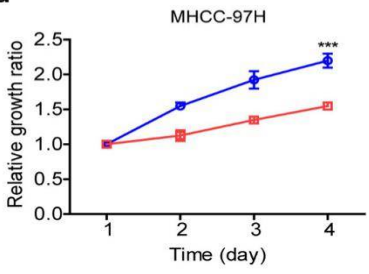

Figure 3: MiR-126 overexpression suppresses HCC cell growth. $(\mathbf{a}, \mathbf{b})$ The growth of HCCLM3 cells and MHCC-97H cells transfected with miR-126 mimics or control was evaluated by CCK-8 assay. O: represents group of miR-NC, $\square$ : represents group of miR-126. (cd) The growth of IRS1-overexpressed or control HCC cells was determined by CCK-8 assay. O: si-NC, $\square$ : si-IRS1; Data are mean \pm SEM; $\left({ }^{*} p<0.05,{ }^{\star *} p<0.01\right.$, $p<0.001)$

\section{MiR-126 sensitizes HCC cells to cisplatin}

To assess the potential impact of miR-126 on HCC cell chemoresistance, miR-126-transfected or sham-transfected HCCLM3 and MHCC-97H cells were treated with cisplatin. As expected, cisplatin treatment apparently reduced the viability of HCC cells in a dose-dependent manner. Notably, miR-126 rendered HCC cells more sensitive to cisplatin treatment in all cisplatin concentrations we examined (Figure 4 a and b). MiR-126 also sensitized HCC cells to cisplatin in a time-dependent manner. Importantly, ectopic expression of IRS1 significantly reversed the effect of miR-126 (Figure $4 \mathrm{c}$ and $\mathrm{d}$ ). Through colony-forming assay, we also observed that miR-126 significantly inhibited the colony-forming capacity of cisplatin treated HCC cells, whereas this inhibition was largely abrogated by the concomitant expression of IRS1 (Figure 4 e). Taken together, miR-126 reduces cisplatin chemoresistance of HCC cells by targeting IRS1. a

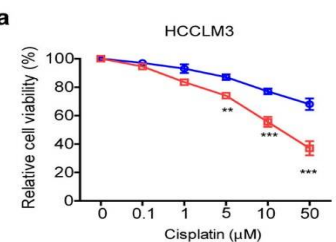

c
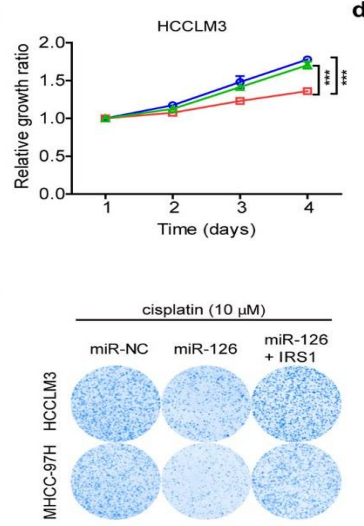

b
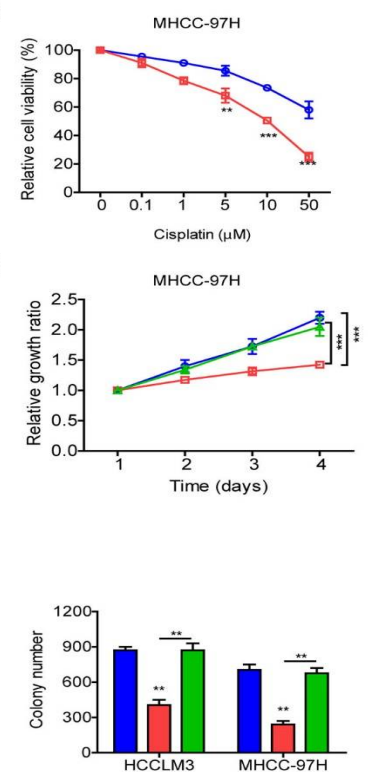

Figure 4: MiR-126 sensitizes HCC cells to cisplatin.(ab) Cell viability was examined in miR-126overexpressed or control HCC cells in the presence of cisplatin(10 $\mu \mathrm{M})$. O: miR-NC, D:miR-126. (c-d) HCC cells were sham-transfected or transfected with miR126 mimics or miR-126 mimics plus IRS1expressingvector, followed by treatment with cisplatin. O: miR-NC + Cis, $\square:$ miR-126 + Cis, $\Delta: m i R-126$ + IRS1 + Cis. Cell proliferation was evaluated by CCK8 assay. (e) HCC cells were sham-transfected or transfected with miR-126 mimics or miR-126 mimics plus IRS1-expressing vector, followed by treatment with cisplatin. Colony formation assay was performed to evaluate cell growth. Data are shown as the mean \pm SEM; $p<0.05, " p<0.01,{ }^{* * * *} p<0.001$ ) 


\section{DISCUSSION}

Emerging evidences have indicated that the dysregulated expression of certain miRNAs can enhance various malignant behaviours of tumor cells, including growth promotion, apoptosis inhibition, triggering epithelial-mesenchymal transition (EMT), or inducing chemoresistance $[8,16]$. Among the cancer-related miRNAs, miR126 has been implicated in inhibiting the metastatic recurrenceof $\mathrm{HCCs}$, suggesting the potential value of miR-126 in the diagnosis or treatment of HCC. However, the impact of miR126 on the growth of HCC cells remains unknown. On the other hand, owning to the fact that cisplatin chemoresistance greatly restrains the therapeutic efficacy of HCC, identifying miRNAs which are involved in the modulation of cisplatin sensitivity in HCC cells is also of great significance.

This study first time demonstrated that miR-126 expression was decreased in primary $\mathrm{HCC}$ tissues relative to tumor-adjacent tissues. Moreover, the reduction of miR-126 expression was associated with poor survival of HCC patients.

Mechanistically, this study identified IRS1 could be directly targeted by miR-126 for downregulation. The reduction of IRS1 was observed both in mRNA and protein levels, suggesting the ability of miR-126 in degradating IRS1 mRNA.IRS1 functions as a key molecule that transmits signals from IGF-1R to downstream pathways such as the PI3K/Akt and MAPK pathways [17]. Overexpression of IRS1 was reported to promote the proliferation of hepatocytes and thus potentially contributed to HCC progression [18,19]. Furthermore, IRS1 reduced the radiosensitivity human prostate cancer cells by accelerating double-strand break repair $[20,21]$.The oncogenic role of IRS1 was also observed in breast cancers [22]. However, how the expression of IRS1 is regulated in human cancers is still far from understood. Here, IRS1 was found to be downregulated by miR126, although miR-126 conferred higher cisplatin chemosensitivity in HCC cells, concomitant overexpression of IRS1 largely abrogated the effect of miR-126. The clinical relevance of miR$126 /$ IRS1 axis was further reinforced by the observation that their expression was negatively correlated in HCC specimens. However, in spite of this present findings in HCC cells, whether miR-126 can also modulate the chemosensitivity in other types of cancer cells still need further investigation. In summary, this study indicates the potential value of miR-126 in the diagnosis or chemotherapy of human hepatocellular carcinoma.

\section{CONCLUSION}

MiR-126 downregulates the proliferation and cisplatin chemoresistance of HCC cells by targeting IRS1.

\section{DECLARATIONS}

\section{Conflict of Interest}

No conflict of interest associated with this work.

\section{Contribution of Authors}

We declare that this work was done by the authors named in this article and all liabilities pertaining to claims relating to the content of this article will be borne by the authors. Lanjuan $\mathrm{Li}$ and Jian Zuo designed all the experiments and revised the paper. Rui Luo, Xiaobin Lou and Chenjie Huang performed the experiments, Jian Zuo wrote the paper.

\section{REFERENCES}

1. Torre LA, Bray F, Siegel RL, Ferlay J, Lortet-Tieulent J, Jemal A. Global cancer statistics, 2012. CA Cancer J Clin 2015; 65: 87-108.

2. Braillon A. Hepatocellular carcinoma. Lancet 2012; 380: 470-461.

3. Park SH, Lee Y, Han SH, Kwon SY, Kwon OS, Kim SS, Kim JH, Park YH, Lee JN, Bang SM, et al. Systemic chemotherapy with doxorubicin, cisplatin and capecitabine for metastatic hepatocellular carcinoma. BMC Cancer 2006; 6: 1-6.

4. Cao $H$, Phan $H$, Yang LX. Improved chemotherapy for hepatocellular carcinoma. Anticancer Res 2012; 32 : 1379-1386.

5. Dasari S, Tchounwou PB. Cisplatin in cancer therapy: molecular mechanisms of action. Eur J Pharmacol 2014; 740: 364-378.

6. Geng $W$, Lo CM, Ng KT, Ling CC, Qi X, Li CX, Zhai $Y$, Liu $X B$, Ma $Y Y$, Man K. Interferon-gamma inducible protein 10 (IP10) induced cisplatin resistance of HCC after liver transplantation through ER stress signaling pathway. Oncotarget 2015; 6: 28042-28056.

7. Alvarez-Garcia I, Miska EA. MicroRNA functions in animal development and human disease. Development 2005; 132: 4653-4662.

8. Sontheimer EJ, Carthew RW. Silence from within: endogenous siRNAs and miRNAs. Cell 2005; 122: 9-12.

9. Calin GA, Croce CM. MicroRNA-cancer connection: the beginning of a new tale. Cancer Res 2006; 66: 73907394. 
10. Callegari E, Elamin BK, Sabbioni S, Gramantieri L, Negrini M. Role of microRNAs in hepatocellular carcinoma: a clinical perspective. Onco Targets Ther 2013; 6: 1167-1178.

11. Chen H, Miao R, Fan J, Han Z, Wu J, Qiu G, Tang H, Peng Z. Decreased expression of miR-126 correlates with metastatic recurrence of hepatocellular carcinoma. Clin Exp Metastasis 2013; 30: 651-658.

12. Available N. World Medical Association Declaration of Helsinki: Ethical Principles for Medical Research Involving Human Subjects. HIV Clinical Trials 2000; 2(1): 92-95.

13. Jeon JH, Kim SK, Kim HJ, Chang J, Ahn CM, Chang YS. Insulin-like growth factor-1 attenuates cisplatin-induced gammaH2AX formation and DNA double-strand breaks repair pathway in non-small cell lung cancer. Cancer Lett 2008; 272: 232-241.

14. Liu MM, Li Z, Han XD, Shi JH, Tu DY, Song W, Zhang J, Qiu XL, Ren Y, Zhen LL. MiR-30e inhibits tumor growth and chemoresistance via targeting IRS1 in Breast Cancer. Sci Rep 2017; 7: 15929-15938.

15. Houghton AM, Rzymkiewicz DM, Ji H, Gregory $A D$, Egea EE, Metz HE, Stolz DB, Land SR, Marconcini LA, Kliment $C R$, et al. Neutrophil elastase-mediated degradation of IRS-1 accelerates lung tumor growth. Nat Med 2010; 16: 219-223.

16. Di Leva G, Garofalo M, Croce CM. MicroRNAs in cancer. Annu Rev Pathol 2014; 9: 287-314.
17. Gorgisen G, Gulacar IM, Ozes ON. The role of insulin receptor substrate (IRS) proteins in oncogenic transformation. Cell Mol Biol (Noisy-le-grand) 2017; 63: 1-5.

18. Nishiyama $M$, Wands JR. Cloning and increased expression of an insulin receptor substrate-1-like gene in human hepatocellular carcinoma. Biochem Biophys Res Commun 1992; 183: 280-285.

19. Tanaka S, Mohr L, Schmidt EV, Sugimachi K, Wands JR. Biological effects of human insulin receptor substrate-1 overexpression in hepatocytes. Hepatol 1997; 26: 598604.

20. Trojanek J, Ho T, Del Valle L, Nowicki M, Wang JY, Lassak A, Peruzzi F, Khalili K, Skorski T, Reiss K. Role of the insulin-like growth factor //insulin receptor substrate 1 axis in Rad51 trafficking and DNA repair by homologous recombination. Mol Cell Biol 2003; 23: 7510-7524.

21. Chitnis MM, Lodhia KA, Aleksic T, Gao S, Protheroe AS, Macaulay VM. IGF-1R inhibition enhances radiosensitivity and delays double-strand break repair by both non-homologous end-joining and homologous recombination. Oncogene 2014; 33: 5262-5273.

22. Surmacz E1, Burgaud JL. Overexpression of insulin receptor substrate 1 (IRS-1) in the human breast cancer cell line MCF-7 induces loss of estrogen requirements for growth and transformation. Clin Cancer Res 1995 ; 1(11):1429-1436. 